\title{
Are the current pain assessment tools used by paramedics in Ireland, suitable for use with cognitively impaired (dementia) patients?
}

\author{
Liam Rooney ${ }^{1,2}$ \\ 1 Advanced Paramedic, Dublin Fire Brigade \\ 2 Graduate Entry Medical School, University of Limerick, Ireland
}

\section{Background}

- Dementia is a disease affecting 55,000 Irish people (1)

- Characterised by progressive cognitive impairment, ranging from mild impairment, which may affect memory, to severe impairment where the ability to communicate may be absent

- These people are at risk of having their pain underassessed and undermanaged (2)

- A survey exploring Irish Paramedics and Advanced Paramedics views on the current pain assessment tools available to them, and whether these tools are suitable for use with dementia patients is proposed

- Existing observational pain assessment tools used with dementia patients are examined and their suitability for pre-hospital use discussed

\section{Introduction}

- Adults with cognitive impairments, such as dementia, are at a much higher risk of not receiving adequate analgesia for their pain (3)

- Estimated between $40 \%$ and $80 \%$ of dementia patients regularly experience pain (4)

- Current pain assessment tools used pre-hospital in Ireland

- Numerical Rating Scale for patients $>8 \mathrm{yrs}$

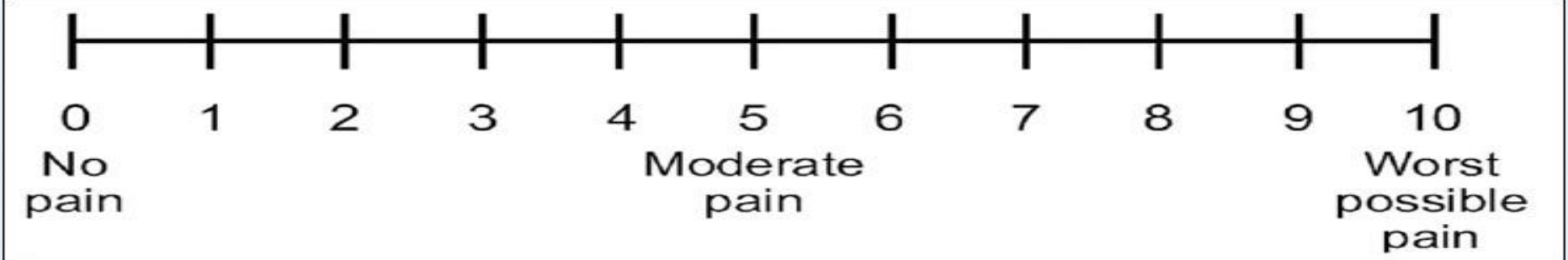

- Wong Baker Scale for pediatric patients

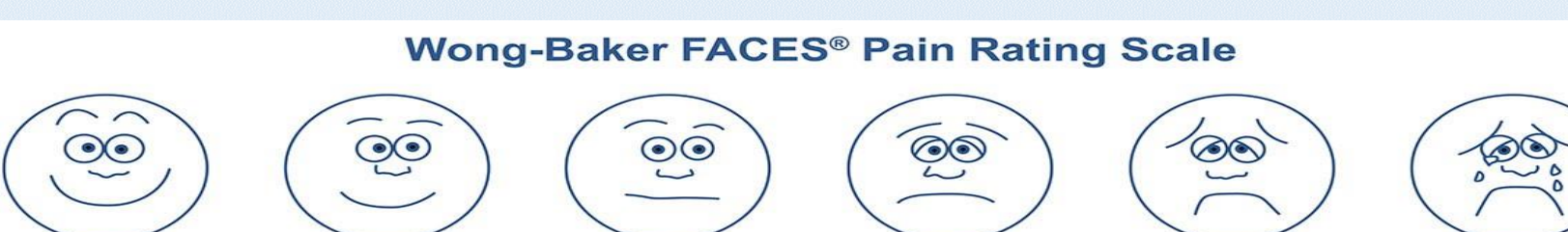

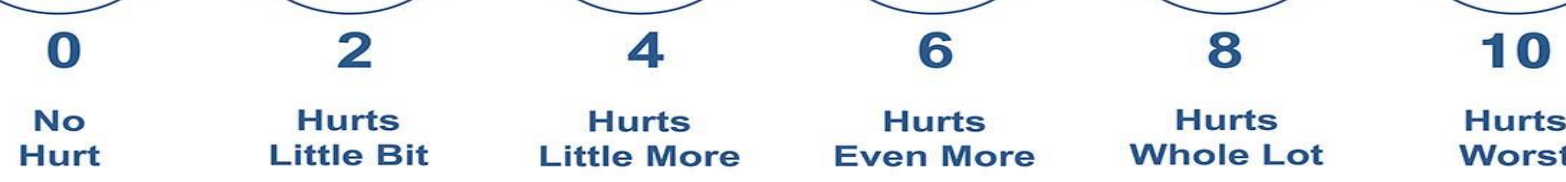

- FLACC Scale for infants

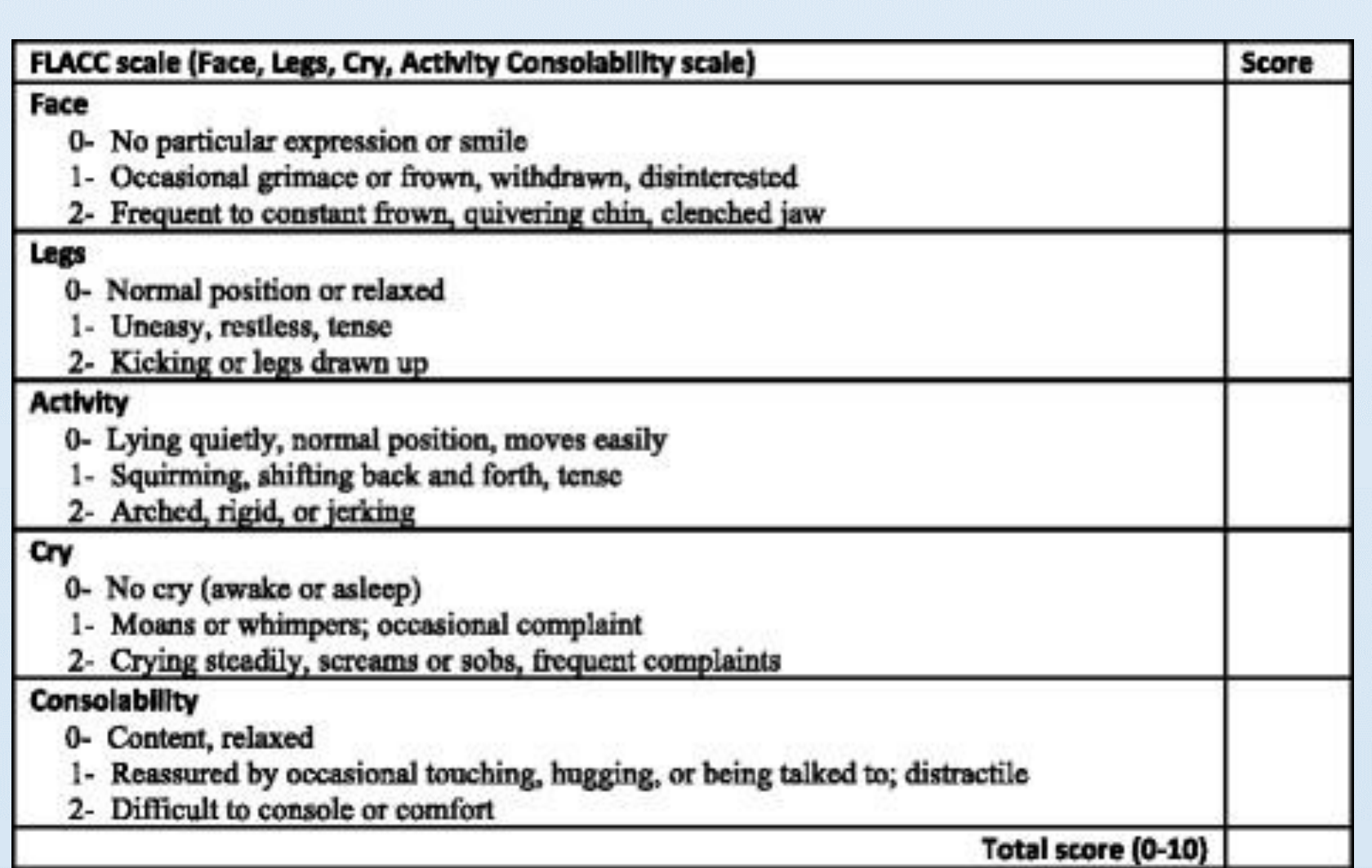

- No specific pain assessment tool for use with patients who are not capable of self-reporting their level of pain

\section{Objective}

- This research aimed to identify observational pain assessment tools used in this cohort. The most consistently recommended tools were identified. The suitability of these tools for use in the pre-hospital setting assessed

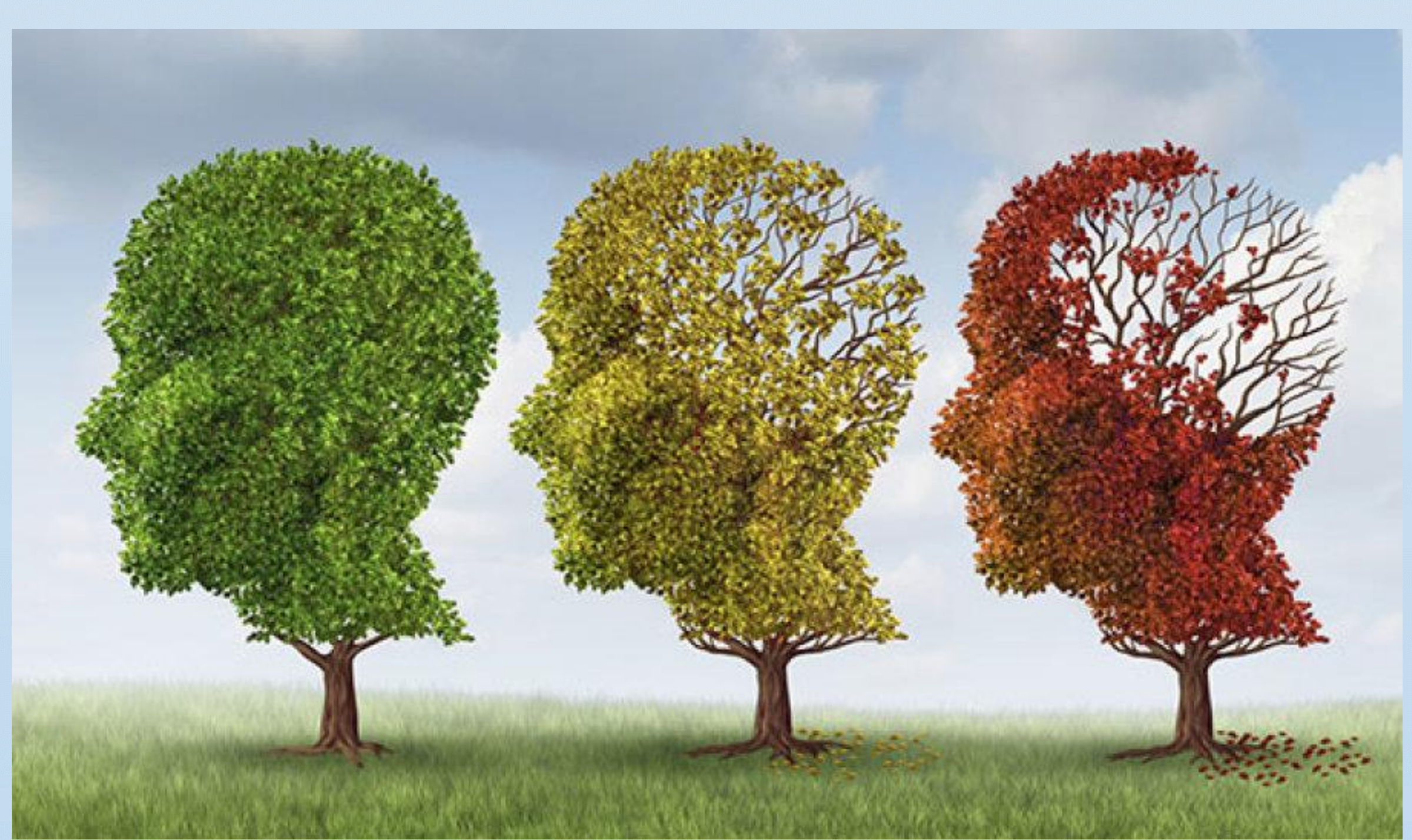

\section{Findings}

- Literature review identified 29 observational pain assessment tools

- Lack of literature relating to pre-hospital setting

- American Geriatric Society (AGS) identified six pain behaviors in dementia patients (5)

- Changes in facial expression, activity patterns, interpersonal relationships and mental status

- Negative vocalisation

- Body language

- These six criteria should be the foundation of any pain assessment too

- Three most consistently recommended tools identified

Abbey Pain Scale

- 6 items assessed

- Meets AGS criteria

- Quick and easy to implement

- Moderate to good reliability and validity (6)

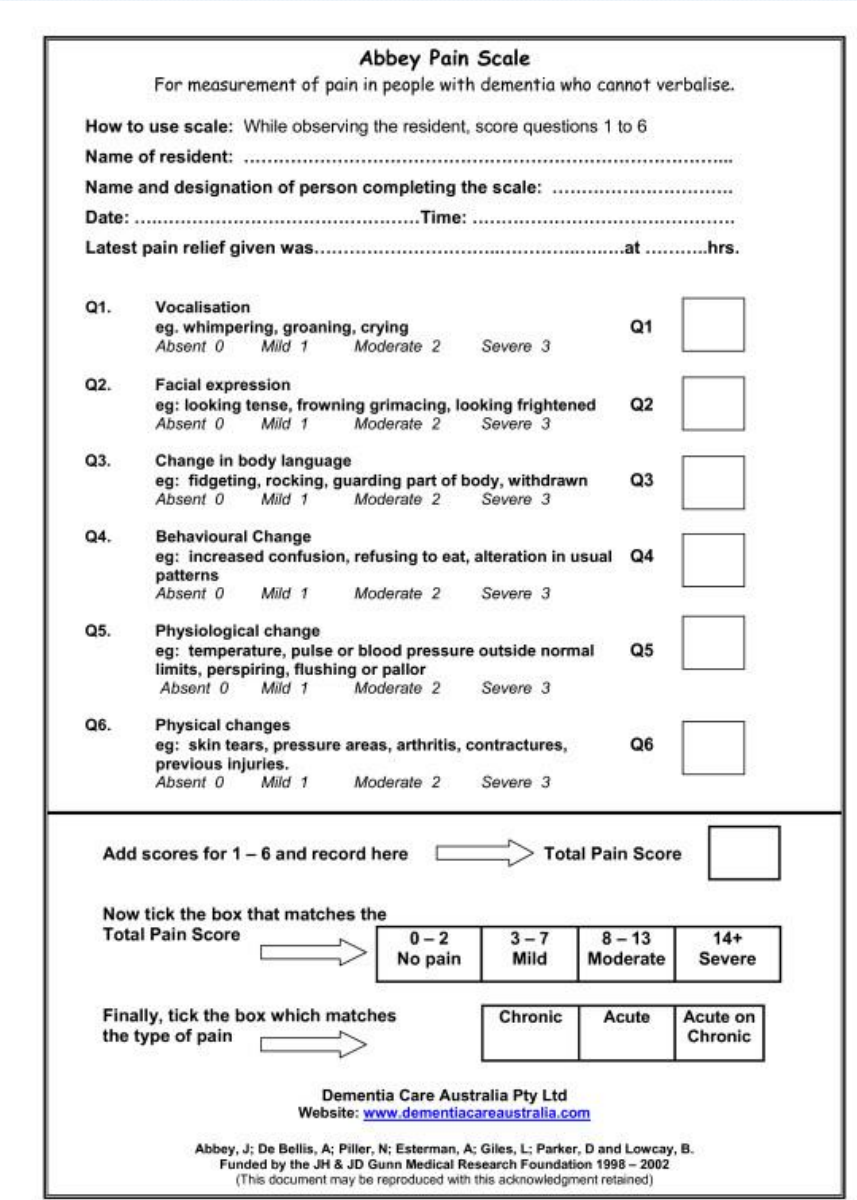

Doloplus 2

- 15 items assessed

- Meets 5 of 6 AGS criteria

- Requires observation over time

- Prior knowledge of patient required

- Moderate to good reliability and validity (6)

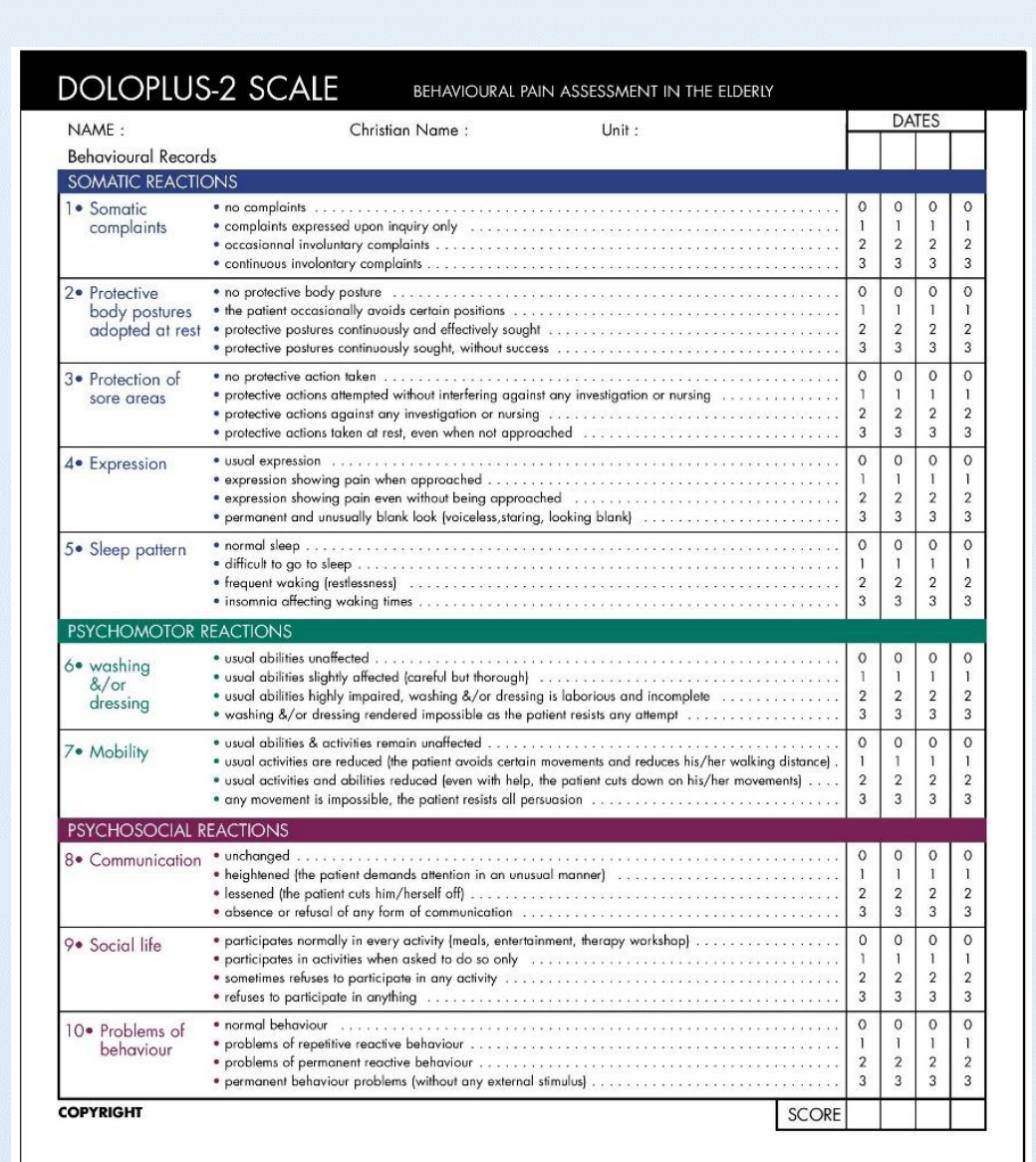

PAINAD

- 5 items assessed

- Meets 3 of 6 AGS criteria

- Less then 5 minutes to implement

- May be influenced by psychological distress

- Good reliability and validity (6)

\section{Conclusion}

- Ability to self report pain is deemed "gold standard"

- Patients with mild to moderate disease, and indeed, some with severe disease, may retain the ability to self report

An observational tool is required when dementia has progressed to the point of being unable to self report or become non-verbal

- It is in these patients where undetected, misinterpreted or inaccurate assessment of pain becomes frequent (7)

Aim of any tool is to gain a good assessment of pain, however, pain scale should be suited to the clinical setting they are being used in

- The feasibility of an assessment tool is an important factor along with reliability and validity

- No one assessment tool could be recommended over another

- Abbey and PAINAD have potential for use pre-hospital, however, further research, clinical evaluation and trial in an ambulance service is required 